\title{
研究通讯
}

\section{Fuzzy 分块矩阵的一个定理}

基于 Fuzzy 矩阵的可乘性分块:

$$
\begin{aligned}
& F=\left[\begin{array}{ll}
F_{p p} & F_{p q} \\
F_{q p} & F_{q q}
\end{array}\right]_{p+q=n}, \\
& G=\left[\begin{array}{ll}
G_{p p} & G_{p q} \\
G_{q p} & G_{q q}
\end{array}\right]_{p+q=n},
\end{aligned}
$$

作者定义了 Fuzzy 分块矩阵的乘法 $\odot$ : $F \bigodot G$

$$
\triangleq\left[\begin{array}{ll}
\left(F_{p p} \circ G_{p p}\right) \cup\left(F_{p q} \circ G_{q p}\right) & \left(F_{p p} \circ G_{p q}\right) \cup\left(F_{p q} \circ G_{q q}\right) \\
\left(F_{q p} \circ G_{p p}\right) \cup\left(F_{q q} \circ G_{q p}\right) & \left(F_{q p} \circ G_{p q}\right) \cup\left(F_{q q} \circ G_{q q}\right)
\end{array}\right],
$$

式 (2) 中的符号 0 是指 Fuzzy 矩阵的乘法运算(或 称合成运算)。

接着,作者证明了关于 Fuzzy 分块矩阵乘法的 一个定理:

定理 1 Fuzzy 矩阵 $F$ 对 $G$ 的分块矩阵乘法 $F \odot G$ 等效于 $F$ 对 $G$ 的乘法 $F \circ G$, 即
$F \odot G \equiv F \circ G$

(3)

或写成

$$
\begin{gathered}
{\left[\begin{array}{ll}
F_{p p} & F_{p q} \\
F_{q p} & F_{q q}
\end{array}\right] \odot\left[\begin{array}{ll}
G_{p q} & G_{p q} \\
G_{q,} & G_{q q}
\end{array}\right]_{p+q=n}} \\
\equiv\left(f_{i k}\right)_{n \times l} \circ\left(g_{k j}\right)_{i \times n} .
\end{gathered}
$$

这个定理应用于下面的特例是格外有价值的：

若

$$
F=G=\left[\begin{array}{ll}
F_{p l} & O_{p q} \\
O_{q L} & F_{q q}
\end{array}\right]_{p+q=n},
$$

即得到

$$
F^{2}=F \odot F=\left[\begin{array}{cc}
\left(F_{p p}\right)^{2} & O_{p q} \\
O_{q p} & \left(F_{q q}\right)^{2}
\end{array}\right],
$$

式中的 $O_{p \times q}, O_{q \times p}$ 分别为 $p \times q 、 q \times p$ 的零矩阵.

涂象初涂承媛

(北京工业大学三系)

\section{循 环 差 集 的 额 外 乘 数*}

H. B. Mann 首先研究了 $(v, k, \lambda)$ 循环差集的额 外乘数一一非 $n=k-\lambda$ 因数的乘数, 并得到了整 数 2 不是任一非平凡循环差集的额外乘数这一有趣 的结果. 本文证明了 Mann (无 Some Theorems on Difference Sets, Canadian J. Math, 1952, 222226) 的一个定理的逆,得到

定理 1 索数 $p$ 是一 $(v, k, \lambda)$ 循环差集 $D$ 的 额外乘数当且仅当

$$
\theta^{p-1}(x) \equiv x^{s}, \quad \bmod d\left(p, x^{v}-1\right)
$$

或

$$
\theta^{p-1}(x) \equiv x^{s}-T(x), \bmod d\left(p, x^{v}-1\right)
$$

对某一整数成立，且 (1) 式成立时 $p \nmid k ，(2)$ 式成 立时 $p \mid k$.

* 国家教委搏士点基金资助的课题.

第 18 期
我们还证明了

定理 2 一奇素数 $p$ 是一 $(v, k, \lambda)$ 循环差集 的额外乘数当且仅当

$$
\begin{gathered}
n^{\frac{p-1}{2}} \theta^{\frac{p-1}{2}}(x)+\frac{1}{\nu} k^{\frac{p-1}{2}}\left(1-n^{\frac{p-1}{2}}\right) T(x) \\
\equiv x^{s} \theta^{\frac{p-1}{2}}\left(x^{-1}\right) \bmod d\left(p, x^{\nu}-1\right)
\end{gathered}
$$

对某一整数 $s$ 成立.

上面的 (1)，(2)、(3) 式中的 $\theta(x)$ 是 $D$ 的 Hall 多项式, 而

$T(x) \equiv 1+x+\cdots+x^{v-1} \bmod \left(x^{v}-1\right)$.

利用定理 2 , 我们证明了

定理 3 整数 3 不是任一非平凡循环差集的 额外乘数。也就是说, 如果 3 是一非平凡的 $(\nu, k, \lambda)$ 循环差集的乘数, 则 $3 \mid n=k-\lambda$.

科学 通 报 